\title{
Myelinated Nerve Fibre Analysis of the Human Small Splanchnic Nerve
}

\author{
By \\ W. TANG, N. GOTO, J. TANAKA and N. OTSUKA \\ Department of Anatomy, Showa University School of Medicine, 5-8, Hatanodai 1, Shinagawa-ku, Tokyo 142, Japan \\ - Received for Publication, May 30, 1997 -

\begin{abstract}
Key Words: Splanchnic nerve, Nerve fiber, Axon, Morphometry, Autonomic nerve
Summary: The aim of the present study is to analyse nerve fibres of the human small splanchnic nerve in relation to the ageing process. The analysis was conducted with the use of a new staining method that makes it possible to discriminate various structures of the nervous tissue. An image-analysing digitiser, a microscope with a drawing tube and a personal computer for storing data and performing statistical analyses were employed in this study. We examined 30 human small splanchnic nerves which were taken from cadaver specimens (20 males and 10 females) aged from 44 to 96 years. Our report may provide a first information concerning the ageing process of the human small splanchnic nerve. The results indicate that a decrease in transverse area and perimeter of myelinated axons in one of the important changes occurring in the human small splanchnic nerve with the ageing process.
\end{abstract}

The autonomic nervous system affects the functions of all other systems and influences human behaviour. It is not surprising, therefore, that clinical manifestations of any illness are influenced by the autonomic nervous system which also affects interactions of individuals with their surroundings ${ }^{1,6}$. As the nervous system is the most important one in the human body, changes with age in this particular system should be studied in detail. Up to now, several studies on the splanchnic nerve have been performed $^{7,10}$. However, to our knowledge, the age-related changes in the human small splanchnic nerve have not been studied yet. It is our purpose in the present study to bring age-related changes to light through analysis of numbers, transverse areas and perimeters of myelinated axons of the human small splanchnic nerve.

\section{Material and Methods}

Small sections of the splanchnic nerve situated near the diaphragm were removed from 30 human cadavers (20 males and 10 females) aged 44-96 years (average age: 70 years). The causes of death had no direct or indirect connection with the central or peripheral nervous system, so the small splanchnic nerves were considered to be normal.

\section{Fixation}

The fixation procedures consisted of two steps as follows: (1) Primary fixation: the material was kept in $3.7 \%$ formaldehyde for at least one week. (2) Secondary fixation: the material was immersed in a mixture of $5 \%$ potassium dichromate and $5 \%$ potassium chromate (1:4 in volume) for three weeks (two weeks at room temperature, and another week at $37^{\circ} \mathrm{C}$ ). The volume of fixative was at least ten times the volume of the specimen.

\section{Washing}

After the fixation, tissue blocks fitted with a nylon-mesh bag or a plastic tissue-basket were washed in running water with a siphon-operated automatic pipette washer for several hours.

\section{Dehydration and celloidin embedding}

The tissue blocks were dehydrated with ethanol at $50 \%, 70 \%, 90 \%, 95 \%$ and pure, and then were embedded in celloidin. The shrinkage of nerve tissue sections during fixation and embedding amounted to approximately $10 \%$ in length. 


\section{Sectioning}

The blocks were sectioned transversely into $20 \mu \mathrm{m}$ thick slices.

\section{Staining}

The sections were stained with LPH triple stain (Luxol fast blue-periodic acid-Schiff-hematoxylin staining). For more details, see the reference section (Goto, 1987).

\section{Morphometry}

The systemic sampling method was employed for the measurement of axons. A sampling site at the centre of the small splanchnic nerve was selected, as shown in Fig. 1. Highly enlarged images $(1,600$ times with oil immersion) of a square eyepiece grid were selected to count the myelinated axons and to measure the transverse area and perimeter of axons: the area covered was $0.016 \times 0.016 \mathrm{~mm}^{2}$. We employed (a) a microscope $\left(\mathrm{BH}_{2}\right.$, Olympus, Tokyo, Japan), equipped with a drawing tube $\left(\mathrm{BH}_{2}-\mathrm{DA}\right.$, Olympus, Tokyo, Japan), (b) a digitiser (Crystizer a KC 3300, Graphtec, Yokohama, Japan) and an analysing system software (original programming), and (c) a computer (PC-9801VX, NEC, Tokyo, Japan) for storing data on-line, calculation and statistical analysis.

We present the data from every decade age group as the mean value \pm SEM (Standard error of mean). The data were statistically examined by analysis of variance (ANOVA). We use the linear regression analysis, in order to determine the relationships between age and number, average area, total area, average perimeter and total perimeter of axons respectively (Scheffé's multiple comparison test). The significance in the present study (except

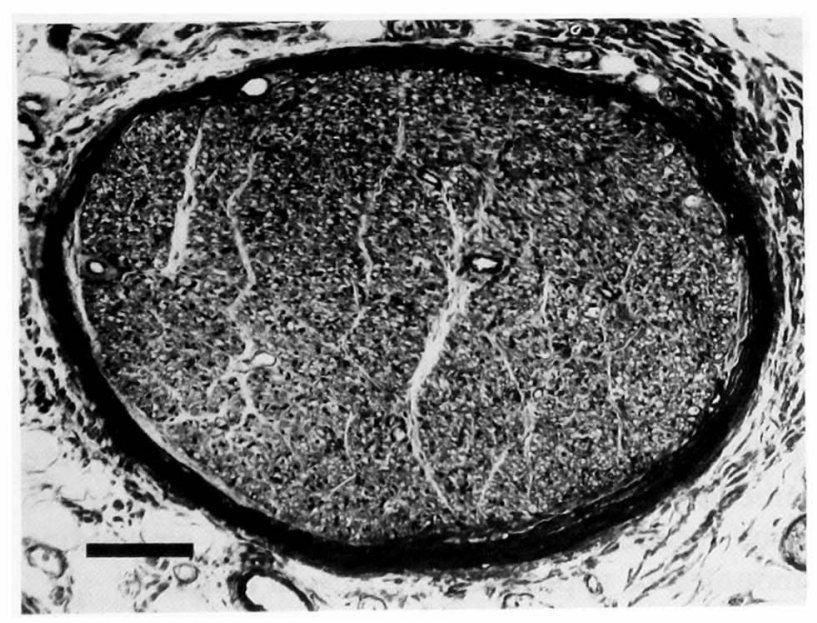

Fig. 1. Low-power view of the small splanchnic nerve of 72 year-old man, LPH stain. Scale bar $=100 \mu \mathrm{m}$ for numbers) was assumed at the level of $p<0.001$ for statistical analysis.

\section{Results}

\section{Numbers of myelinated axons}

We estimated the total number of myelinated axons in the human small splanchnic nerve as being between 4 and 34 (average 13.63) within a unit area of $0.016 \times 0.016 \mathrm{~mm}^{2}$. The data for all cases are listed in Table 1. The myelinated nerve fibres appeared as a blue-green myelin sheath surrounding a dark purple or black axon (Fig. 2). According to our data, the number of myelinated axons did not show a marked change with age $(r=-0.17$, $p>0.10)$.

\section{Transverse areas of myelinated axons}

The transverse area of myelinated axons in the human small splanchnic nerve ranged from 0.39 to 3.36 (average 1.60) $\mu \mathrm{m}^{2}$. The average transverse area of myelinated axons was smaller in the 90-99 year age group, 80-89 group, 70-79 group and 6069 group than that in the $40-59$ group, $(P<0.001)$ : $27.0 \%, 49.8 \%, 30.9 \%$ and $26.6 \%$ of the area in the 40-59 group, respectively (Table 2). A scatter diagram with the regression line between the average transverse area and age is shown in Fig. 3. It reveals that the average area of myelinated axons in the transverse section is reduced with age $(\mathrm{r}=-0.56$, $P<0.001)$. The total transverse area also decreases with age ( $r=-0.67, P<0.001$, Fig. 4$)$. In the 90-99 and 80-89 groups, the myelinated axons were smaller and thinner compared with those in the 40 49 group (Table 3 ).

\section{Perimeter of myelinated axons}

The average perimeter of myelinated axons in the human small splanchnic nerve was $3.50 \mu \mathrm{m}$ (ranging from 1.43 to 5.81 ). The average perimeter of myelinated axons was reduced with age $(\mathrm{r}=-0.54, \mathrm{P}<0.001$, Fig. 5). It is obvious that there is also a negative correlation between age and the total perimeter of myelinated axons $(r=-0.51$, $P<0.05$, Fig. 6).

\section{Discussion}

It is known that the autonomic nervous system is the orchestrator for adaptive responses to stress from the emotional, environmental or sociologic point of view. Although the human splanchnic nerves supply the viscera, the small splanchnic nerve is considered to affect the function of the su- 
Table 1. Fibre analysis of the small splanchnic nerve

\begin{tabular}{|c|c|c|c|c|c|c|c|}
\hline Subject & Age & Sex & NAx & MAAx & TAAx & MPAx & TPAx \\
\hline 1395 & 44 & $\mathbf{F}$ & 7 & 3.63 & 23.50 & 5.81 & 40.70 \\
\hline 1393 & 45 & $\mathbf{M}$ & 17 & 1.53 & 26.10 & 3.63 & 61.80 \\
\hline 1385 & 54 & $\mathbf{M}$ & 13 & 1.59 & 20.60 & 3.32 & 43.20 \\
\hline 1371 & 57 & $\mathbf{M}$ & 16 & 2.70 & 45.60 & 4.90 & 78.10 \\
\hline 1369 & 58 & $\mathbf{M}$ & 9 & 2.10 & 18.80 & 4.30 & 38.30 \\
\hline 1409 & 59 & $\mathbf{M}$ & 8 & 2.40 & 19.50 & 4.70 & 37.50 \\
\hline 1427 & 60 & $\mathbf{F}$ & 12 & 2.60 & 30.80 & 4.60 & 54.80 \\
\hline 1416 & 64 & F & 12 & 2.30 & 27.30 & 4.30 & 51.30 \\
\hline 1404 & 65 & $\mathbf{M}$ & 10 & 2.10 & 21.50 & 4.20 & 41.70 \\
\hline 1378 & 66 & $\mathbf{F}$ & 21 & 1.40 & 29.20 & 3.50 & 72.70 \\
\hline 1412 & 67 & $\mathbf{M}$ & 34 & 0.73 & 24.90 & 2.20 & 75.60 \\
\hline 1392 & 69 & $\mathbf{M}$ & 29 & 1.10 & 30.80 & 2.70 & 79.00 \\
\hline 1382 & 70 & $\mathbf{M}$ & 15 & 2.20 & 32.90 & 4.20 & 63.30 \\
\hline 1372 & 73 & $\mathbf{F}$ & 14 & 1.10 & 15.90 & 3.00 & 42.10 \\
\hline 1389 & 75 & $\mathbf{M}$ & 11 & 1.60 & 17.70 & 3.20 & 34.90 \\
\hline 1377 & 77 & $\mathbf{M}$ & 11 & 1.80 & 19.90 & 4.20 & 46.20 \\
\hline 1390 & 77 & $\mathbf{M}$ & 9 & 1.06 & 9.53 & 2.74 & 24.64 \\
\hline 1364 & 77 & $\mathbf{F}$ & 17 & 0.95 & 16.20 & 2.60 & 44.18 \\
\hline 1414 & 78 & $\mathbf{M}$ & 16 & 1.40 & 21.80 & 3.20 & 51.60 \\
\hline 1394 & 81 & $\mathbf{M}$ & 22 & 1.40 & 30.20 & 3.30 & 73.60 \\
\hline 1405 & 82 & $\mathbf{M}$ & 9 & 0.99 & 8.96 & 2.80 & 25.01 \\
\hline 1375 & 83 & $\mathbf{M}$ & 19 & 0.39 & 7.34 & 1.43 & 27.10 \\
\hline 1407 & 84 & $\mathbf{M}$ & 9 & 1.10 & 9.98 & 2.90 & 25.80 \\
\hline 1397 & 84 & $\mathrm{~F}$ & 17 & 0.60 & 10.50 & 2.20 & 37.40 \\
\hline 1368 & 85 & $\mathbf{M}$ & 8 & 2.30 & 18.30 & 4.30 & 34.40 \\
\hline 1418 & 85 & $\mathbf{M}$ & 15 & 0.77 & 11.60 & 2.20 & 33.60 \\
\hline 1410 & 86 & $\mathbf{F}$ & 5 & 0.60 & 3.20 & 2.20 & 10.90 \\
\hline 1383 & 87 & $\mathrm{~F}$ & 10 & 1.50 & 15.20 & 3.40 & 34.40 \\
\hline 1415 & 91 & $\mathbf{M}$ & 8 & 1.80 & 14.60 & 3.70 & 29.50 \\
\hline 1403 & 96 & $\mathrm{~F}$ & 4 & 1.60 & 6.40 & 3.70 & 15.50 \\
\hline Mean & 72 & & 13.63 & 1.59 & 20.73 & 3.50 & 45.01 \\
\hline SD & 13.11 & & 6.52 & 0.70 & 10.00 & 0.96 & 17.88 \\
\hline
\end{tabular}

NAx: number of axons in the left small splanchnic nerve, MAAx: mean area of axons,

TAAx: total area of axons, MPAx: mean perimeter of axons, TPAx: total perimeter of axons.

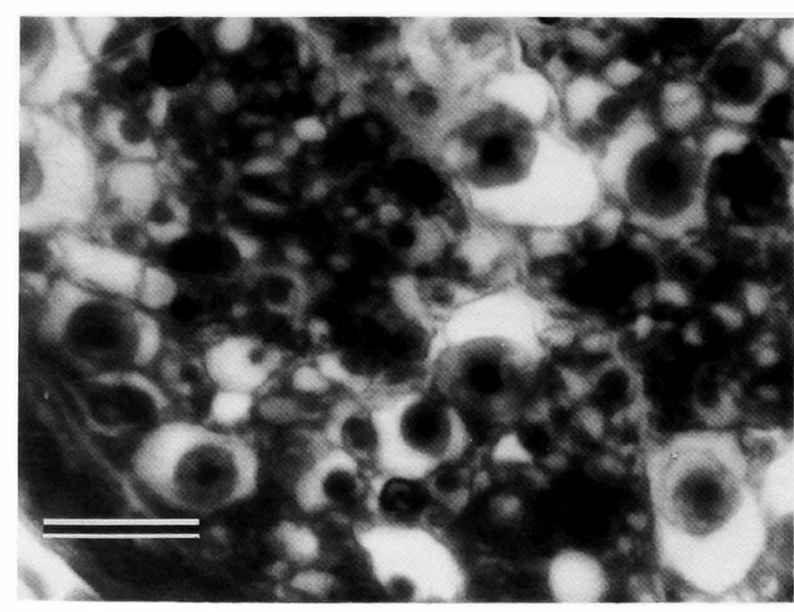

Fig. 2. High-power view of the small splanchnic nerve of 60 year-old woman, LPH stain. Axons are stained in dark purple or black, and surrounded by myelin sheath. Scale bar $=0.1 \mu \mathrm{m}$ prarenal body and the kidney through the ramus renalis. Up to now, there have been few reports available on the morphometry of nerve fibres in the human small splanchnic nerve ${ }^{11)}$ - especially with regard to axons. The reason may be due to a lack of discriminative staining method for nerve fibres, and of modern methods for morphometric analyses. For example, various silver impregnation methods have been used for the staining of the nervous system, but they cannot be used for morphometric analyses, except for numbers, and this for two reasons: shrinkage and discrimination. We employed a new staining method, the LPH staining method ${ }^{4}$. The result indicates that this method greatly facilitates morphometric studies on the peripheral nervous system thanks to the clear discrimination of structures, for example axons (blue or dark purple), myelin sheaths (blue-green), neuronal nucleoli (dark blue), other cell nuclei (colour similar to conventional hematoxylin stains), connective tissue 
Table 2. Mean area of the small splanchnic nerve

\begin{tabular}{lcccc}
\hline Age group & No. & Average age & Mean area & Atrophy rate \\
\hline $40-49$ & 2 & 44.5 & 2.45 & - \\
$50-59$ & 4 & 57.0 & 2.20 & - \\
\hline $60-69$ & 6 & 65.2 & 1.71 & $26.61 \%$ \\
$70-79$ & 7 & 75.3 & 1.61 & $30.90 \%$ \\
$80-89$ & 9 & 84.1 & 1.17 & $49.79 \%$ \\
$90-99$ & 2 & 93.5 & 1.70 & $27.04 \%$ \\
\hline
\end{tabular}

The atrophy rate is a percentile value of the mean area to that of the $40-59$ years group.

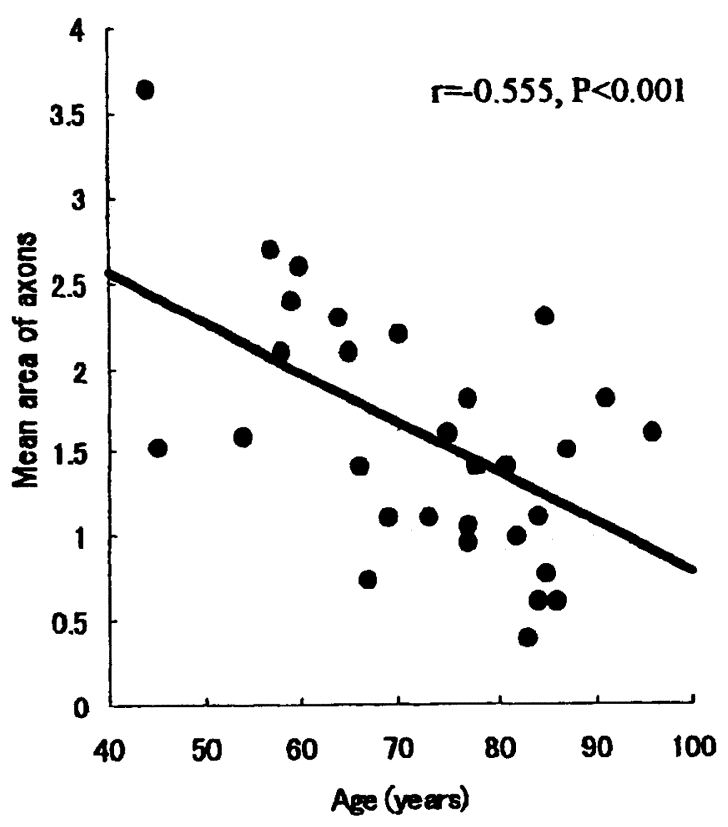

Fig. 3. Scatter diagram and regression line showing the correlation between the age and the reduction of mean area of axons in the small splanchnic nerve.

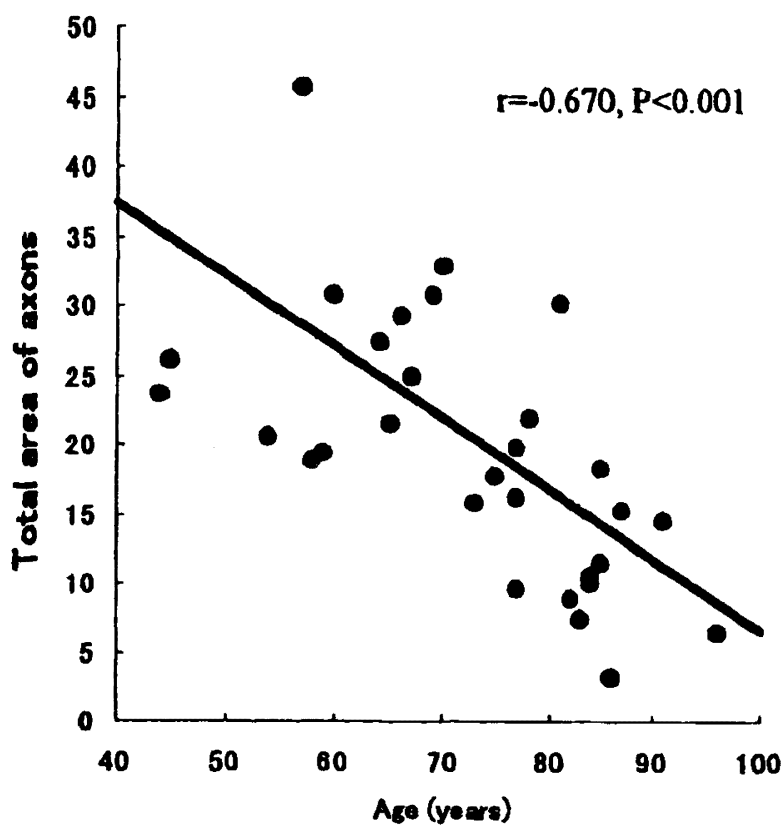

Fig. 4. Scatter diagram and regression line showing the correlation between the age and the reduction of total area of axons in the small splanchnic nerve.

Table 3. Total area of the small splanchnic nerve

\begin{tabular}{lcccc}
\hline Age group & No. & Average age & Total area & Atrophy rate \\
\hline $40-49$ & 2 & 44.5 & 24.80 & - \\
$50-59$ & 4 & 57.0 & 37.98 & - \\
\hline $60-69$ & 6 & 65.2 & 27.40 & $12.65 \%$ \\
$70-79$ & 7 & 75.3 & 20.62 & $34.31 \%$ \\
$80-89$ & 9 & 84.1 & 11.12 & $64.58 \%$ \\
$90-99$ & 2 & 93.5 & 10.50 & $66.55 \%$ \\
\hline
\end{tabular}

The atrophy rate is a percentile value of the total area to that of the $40-59$ years group. 


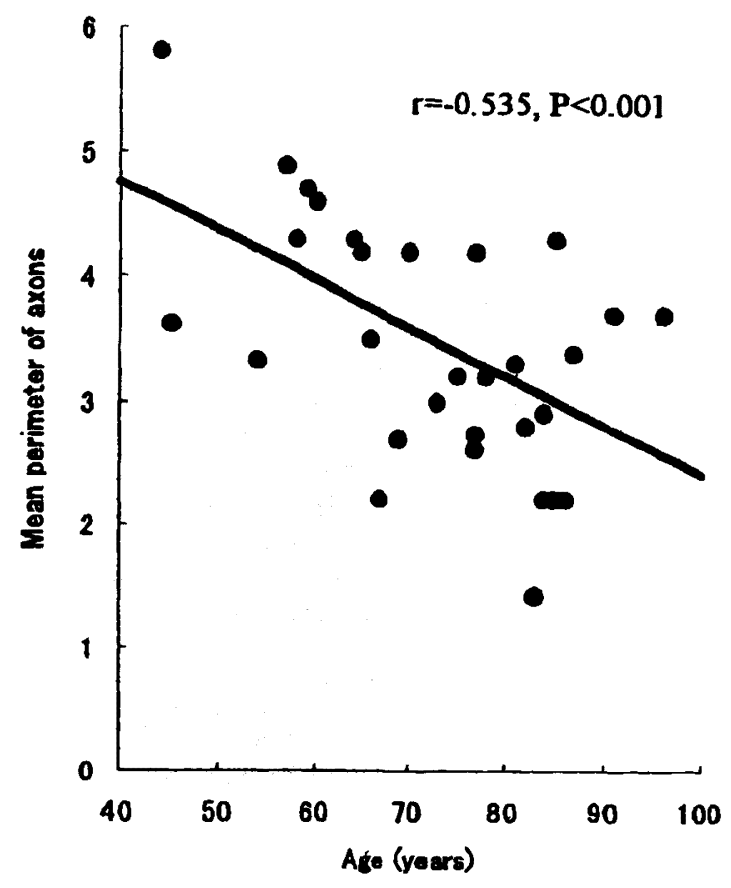

Fig. 5. Scatter diagram and regression line showing the correlation between the age and the reduction of mean perimeter of axons in the small splanchnic nerve.

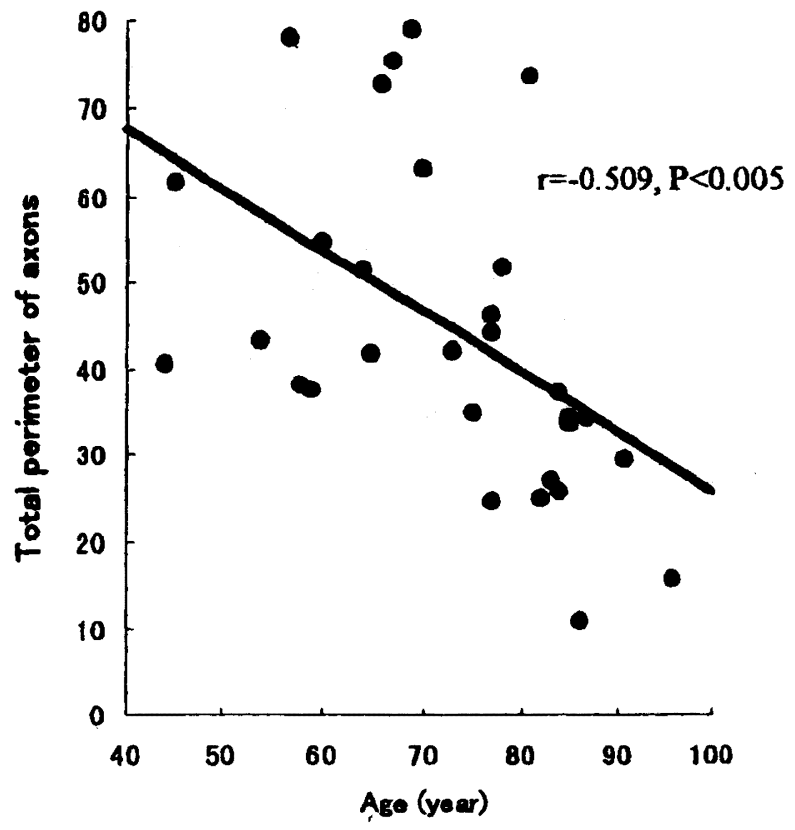

Fig. 6. Scatter diagram and regression line showing the correlation between the age and the reduction of total perimeter of axons in the small splanchnic nerve. (faintly pink), amyloid bodies and lipofuscin granules (light purple), etc. The method is also very suitable for morphometric research on the nervous system because of its clear differentiation of structures and minimal shrinkage (only about $10 \%$ in length ${ }^{4}$. The fixation method for the morphometric analysis is one of the most important steps in the methodology. The usual formaldehyde fixation may cause a wide range of tissue shrinkages during histologic processing. In our study, we used the secondary fixation with chromic acid after formaldehyde fixation in order to obtain better staining results and to minimize tissue shrinkage ${ }^{4,5}$ ).

The next point is the embedding method. For light microscopy, there exist at present two main embedding methods: paraffin and celloidin. The marked shrinkage of tissue that occurs during processing is one of the disadvantages of using paraffin. The paraffin technique is therefore not suitable for morphometric analysis. It was replaced in the present study by the celloidin method after secondary fixation with chromic acid ${ }^{4}$.

As for the microscopic morphometry, a combination of a microscope, drawing tube, digitiser and computer has made this kind of research both easy and accurate, as is described in detail in the "Material and Methods" section above. Otherwise, it would be very difficult or even impossible to measure the areas of irregular shapes with any degree of accuracy.

Several studies about age-related changes in various nerves have been reported since the LPH staining method was introduced. For example, Fujii et al. reported that the transverse areas and circularity ratios of the axons in the facial nerve do not change with age, but the numbers of axons become reduced with age $(r=-0.59)^{2)}$. In another article, the same author reported that the transverse axonal areas of the cochlear nerve is reduced with age $(\mathrm{r}=-0.87, \mathrm{p}<0.01)$, while the number and transverse areas of amyloid bodies in the vestibulocochlear nerve increase with age (area: $r=0.76$, $\mathrm{p}<0.01$; number: $\mathrm{r}=0.77, \mathrm{p}<0.01$ ). The number of vestibular nerve fibres do not show any change with age ${ }^{3)}$. In Zhang et al. ${ }^{14)}, 38$ human spinal cords aged from 41 to 97 years were used to count the myelinated axons and to measure the axonal transverse area of the posterior funiculus at C6 level. This research revealed that a decrease in size and number of axons was one of the impotant changes occurring with the ageing process in the human spinal posterior funiculus (number: $r=-0.85, p<0.001$; area: $r=-0.77, p<0.001)$. As to the human deep peroneal nerve, Yanagisawa et $a .^{13)}$ reported the number and transverse area of axons measured in 21 males whose ages ranged from 41 to 89 years. 
The results indicate that a slenderisation and diminution of axons, and especially a disappearance of large axons are the main picture of the ageing process of the deep peroneal nerve (number: $\mathrm{r}=-0.82, \mathrm{p}<0.01$; area: $\mathrm{r}=-0.75, \mathrm{p}<0.01)$.

In the clinical field, Fujii et al. ${ }^{3)}$ suggested that a presbyacusis might be due to a reduction of the axonal area of the cochlear nerve with age. Zhang et al. ${ }^{14)}$ found that an atrophy and a decrease in number of myelinated axons were the main changes connected with ageing occurring in the human spinal cord. However, the clinical appearance of the small splanchnic nerve in relation to the ageing process still remains unknown and should be elucidated in future.

As for the regression lines between age and numbers of axons, ageing processes of several nerves were analyzed and evaluated: the facial nerve $^{2)}$, the vestibulocochlear nerve ${ }^{3)}$, the posterior funiculus of the spinal cord $^{14)}$, the deep peroneal nerve $^{13)}$ and the oculomotor nerve ${ }^{12)}$. Furthermore, it can be concluded that changes with the ageing process are more evident in motor nerve fibres than in sympathetic nerve fibres. In the present study, we found that the number of myelinated fibres in the small splanchnic nerve remained almost constant with age, while the axonal areas and perimeters decreased with age (mean area: $\mathrm{r}=-0.55, \mathrm{p}<0.001)$. The results indicate that the vulnerability of the small splanchnic nerve may be related to ageing. It is considered that there may be a correlation between ageing and the visceral hypofunction from a morphometric point of view.

\section{Acknowledgments}

Preliminary results of this study were presented at the 101th Congress of the Japanese Association of Anatomists, Fukuoka, Japan, 1996. The authors would like to thank Profs. T. Kimura and K. Shimada of the Department of Anatomy, Showa University School of Medicine, for their encouragement and support during this research, Dr. M.
Suzuki for computer analyses and Mr. M. Shibata for technical assistance.

\section{References}

1) Appenzeller O. Clinical autonomic failure, practical concepts. Elsevier, Amsterdam - New York - Oxford, 1986; 1-20.

2) Fujii $M$ and Goto $N$. Nerve fiber analysis of the facial nerve. Ann Otol Rhinol Laryngol 1989; 98:732-735.

3) Fujii $\mathbf{M}$, Goto $\mathbf{N}$ and Kikuchi $\mathbf{K}$. Nerve fiber analysis and the aging process of the vestibulocochlear nerve. Ann Otol Rhinol Laryngol 1990; 99:863-870.

4) Goto N. Discriminative staining methods for the nervous system: Luxol fast blue-periodic acid-Schiff-hematoxylin triple stain and subsidiary staining methods. Stain Technol 1987; 62:305-315.

5) Goto $N$ and Kaneko $M$. Olivary enlargement: chronologic and morphometric analysis. Acta Neuropathol 1981; 54: 275-282.

6) Horiguchi $M$, Kida $M$ and Kodama $K$. Anatomy of the peripheral nerves - basis and development. Science Communications International 1995; 93-115 (in Japanese).

7) Isomura G, Kanematsu $M$ and Shimizu N. Degeneration of the greater splanchnic nerve by operation. Anat Anz 1985; 158:209-216.

8) Isomura G, Iwata S, Chiba $M$ and Shimizu N. Constitution of the greater splanchnic nerve in the rat. Anat Anz 1985; 159:159-171.

9) Kuo DC, Yang GC, Yamasaki DS and Krauthamer GM. A wide field electron microscopic analysis of the fiber constituents of the major splanchnic nerve in cat. J Comp Neurol 1982; 210:49-58.

10) Niijima A. Electrophysiological study on nervous pathway from splanchnic nerve to vagus nerve in rat. Am J Physiol 1983; 244:R888-890.

11) Takeshige $Y$, Miyazaki $M$, Hata $Y$, Sato $S$ und Hoshii $Y$. Morphologische Untersuchung Über den Nervus splanchnicus major und minor. Kurume Igakukai Zasshi 1962; 25: 27-34 (in Japanese).

12) Takeshita $Y$, Matsumoto $K$, Goto $N$ and Shibata $M$. Nerve fiber analysis and aging process of the human oculomotor nerve. Showa Univ J Med Sci 1996; 8:55-61.

13) Yanagisawa K, Goto $N$, Kimura $T$ and Tanaka J. Nerve fiber analysis on aging process of deep peroneal nerve in man. J Showa Med Assoc 1994; 54:249-254 (in Japanese).

14) Zhang $C$, Goto $N$ and Zhou M. Morphometric analyses and aging process of nerve fibers in the human spinal posterior funiculus. Okajimas Folia Anat Jpn 1995; 72:259-264. 